\title{
ERRATUM
}

John F. Cvar • Gunnar Ryge*

\section{Reprint of}

\section{Criteria for the clinical evaluation of dental restorative materials}

Published online: 14 January 2006

C) Springer-Verlag 2006

In the online version of the article, the names of the authors should read:

John F. Cvar · Gunnar Ryge

The names are correct in the printed version.

The online version of the original article can be found at: http://dx.doi.org/10.1007/s00784-005-0018-z

*Authors deceased

Contact: G. Schmalz $(\bowtie)$

Poliklinik für Zahnerhaltung und Parodontologie,

Universität Regensburg,

Franz-Josef-Strau $\beta$-Allee 11,

93042 Regensburg, Germany

e-mail: Gottfried.schmalz@klinik.uni-regensburg.de

Tel.: +49-941-9446024

Fax: +49-941-9446025 